\title{
Anne Bouchy (éd.), Le vivre ensemble à Sasaguri, une commune de Kyūshū. Dans l'entrelacs des dynamiques du dedans et du dehors
}

Paris, Cahiers d'Extrême-Asie nº 22, 2013, 646 p.

Jean-Pierre Albert

\section{(2) OpenEdition \\ Journals}

\section{Édition électronique}

URL : http://journals.openedition.org/assr/28172

DOI : $10.4000 /$ assr.28172

ISSN : $1777-5825$

\section{Éditeur}

Éditions de l'EHESS

Édition imprimée

Date de publication : 31 décembre 2016

Pagination : 274

ISSN : 0335-5985

Référence électronique

Jean-Pierre Albert, « Anne Bouchy (éd.), Le vivre ensemble à Sasaguri, une commune de Kyūshū. Dans l'entrelacs des dynamiques du dedans et du dehors ", Archives de sciences sociales des religions [En ligne], 176 | octobre-décembre 2016, mis en ligne le 13 juillet 2017, consulté le 25 septembre 2020. URL : http://journals.openedition.org/assr/28172 ; DOI : https://doi.org/10.4000/assr.28172

Ce document a été généré automatiquement le 25 septembre 2020.

(c) Archives de sciences sociales des religions 


\title{
Anne Bouchy (éd.), Le vivre ensemble à Sasaguri, une commune de Kyūshū. Dans l'entrelacs des dynamiques du dedans et du dehors
}

\author{
Paris, Cahiers d'Extrême-Asie nº 22, 2013, 646 p.
}

Jean-Pierre Albert

\section{RÉFÉRENCE}

Anne Bouchy (éd.), Le vivre ensemble à Sasaguri, une commune de Kyūshū. Dans

l'entrelacs des dynamiques du dedans et du dehors, Paris, Cahiers d'Extrême-Asie n ${ }^{\circ} 22$, 2013, 646 p.

1 Cette livraison des Cahiers d'Extrême-Asie, revue publiée par l'École Française d'Extrême Orient (site de Kyoto), est presque entièrement consacrée à l'édition des résultats de l'enquête collective conduite par une équipe franco-japonaise d'ethnologues entre 2004 et 2010. Ce dossier, comprenant une introduction de l'éditrice et huit articles, est complété par une bibliographie commentée d'une trentaine de pages présentant des travaux classiques d'ethnologie du Japon en rapport avec la thématique du numéro. Il s'agit là d'une monographie hors norme, tant par son ampleur que par la longue collaboration entre chercheurs qui l'a rendue possible. Il ne fait guère de doute que ce recueil, au-delà de la richesse documentaire d'une monographie, apporte des données de portée plus générale qui intéresseront les historiens et ethnographes du Japon en général. Il offre aussi aux chercheurs français une occasion rare d'avoir accès à la très riche tradition ethnographique japonaise.

2 L'enquête porte sur la commune de Sasaguri, une ville d'environ 30000 habitants située dans le département de Fukuoka à la pointe nord de la grande île de Kyūshū. Son choix tient dans une large mesure à la présence sur son territoire d'un lieu de pèlerinage, très 
connu à l'échelle régionale et même au-delà : le «nouveau pèlerinage de Shikoku à Sasaguri ", c'est-à-dire une réplique d'un des plus grands complexes de lieux sacrés du Japon. Cela suffit à donner à l'analyse des différentes formes de présence du religieux une place importante dans l'enquête. Mais les articles réunis portent également sur d'autres aspects de la vie locale - rapports à l'espace forestier, activité minière aujourd'hui disparue, condition des femmes au temps de la mine, etc. Son fil directeur est la question des relations entre le « dedans » et le « dehors », entendue en particulier comme rencontre de facteurs internes d'évolution et de facteurs externes, liés à des échelles diverses à l'histoire de la région et celle du Japon. On retiendra principalement dans ce compte rendu les travaux consacrés à la vie religieuse à Sasaguri, qui représentent du reste plus de la moitié du volume et sont présents, peu ou prou, dans toutes les contributions.

La première partie de l'ouvrage («Cadre socioculturel et environnement ») s'ouvre par le long article de Suzuki Masataka (« Continuités et transformations de la société locale. Le fait coutumier dans le village de Wakasugi », 87 p.) qui se présente comme une monographie très précise du village de Wakasugi (un des 21 quartiers de la commune de Sasaguri) dont sont étudiés principalement les aspects religieux : investissement religieux de l'espace, récits de fondation des temples, évolution des dévotions, etc. L'accent est mis à la fois sur les continuités et les changements (introduction du kagura - une danse théâtrale rituelle -, nouvelle économie du funéraire, place du tourisme). Cette étude mobilise une érudition considérable et, outre l'intérêt des analyses présentées, elle est appelée à constituer une source documentaire incontournable sur l'histoire religieuse de la région.

4 La contribution d'Anne Bouchy qui vient ensuite, «Les rapports communautaires aux espaces forestiers entre politiques du dehors et stratégies du dedans. Les montagnesforêts de Sasaguri » (p. 115-202), semble moins directement consacrée à la dimension religieuse que le reste du dossier. Après une mise en contexte de l'étude, l'auteur développe en effet l'historique des différents types de forêts existant sur le territoire et le met en relation avec les différentes modalités d'exploitation ou de gestion qui leur correspondent en insistant à la fois sur les facteurs internes et les facteurs externes d'évolution. Mais elle précise également en quoi cet environnement est une ressource pour différents investissements religieux. La forêt est en effet devenue le cadre d'une "thérapie forestière " dont on peut penser qu'elle est «une nouvelle forme d'un religieux diffus associée à des modalités renouvelées des pratiques du pèlerinage ». Elle montre également comment, dans la gestion de conflits ou problèmes locaux, dans laquelle interviennent des instances régionales, voire nationales, le religieux a pu être un instrument efficace de résolution. En cela, elle tente de faire le lien entre les approches du rapport au monde naturel et celles du religieux, deux domaines traités séparément en général.

5 La seconde partie de la revue, intitulée " Texture du religieux ", réunit quatre articles. Le premier est celui de Mori Hiroko : «Le mont Wakasugi, son sanctuaire et le shugen dans l'histoire de Kyūshū et de l'Asie de l'Est ». Il se compose de deux grandes parties, la première consacrée à l'historique des cultes associés au mont Wakasugi dans la longue durée, la seconde au sanctuaire d'Ishii-bô et à ses liens avec le shugen. Il vaut en particulier par l'ampleur de l'érudition mobilisée, à partir de laquelle l'auteur parvient à reconstituer l'histoire complexe et tourmentée des lieux. 
6 La contribution de Nakayama Kazuhisa («La dynamique de création, réplication et déclin des lieux de pèlerinage. Le nouveau pèlerinage de Shikoku à Sasaguri ») associe une très solide documentation sur l'histoire et les pratiques actuelles du pèlerinage étudié à des réflexions très intéressantes d'une part sur le sens du pèlerinage pour ceux qui l'accomplissent, d'autre part sur le phénomène de la réplication d'un site de référence. Il se conclut enfin par l'analyse des mutations les plus contemporaines : la part du site naturel dans l'attrait des lieux et la recherche de puissants médiateurs charismatiques.

7 Un second article de Suzuki Masataka ("Modernisation des temples bouddhiques et société locale. Le Nanzō-in de Sasaguri ») est l'étude monographique du temple cité dans le titre qui était d'abord seulement le point de départ du parcours des 88 étapes du pèlerinage de Shikoku. Son histoire récente est marquée par l'installation en 1995 d'une statue géante du Bouddha couché qui a complètement renouvelé les pratiques de dévotion. Un des grands intérêts de cette étude est de présenter la vitalité de ces pratiques et leur constant enrichissement.

8 À la différence des autres articles du dossier, celui de Charlotte Lamotte, « La pierre qui vit. Naissance et mort des statues dans une ville de pèlerinage », se concentre sur un point précis, une énigme trouvée sur le terrain : comment les statues acquièrent-elles une sorte de personnalité aux yeux de leurs fidèles ? La réponse est cherchée à la fois dans la théorie locale de la figuration et des effets du culte, et dans une approche comparative qui permet de mobiliser des outils d'analyse anthropologique.

9 La dernière section de l'ouvrage, «Au cœur du social », aborde la question du religieux du quotidien. Ainsi l'étude d'Ishikawa Toshiko, «Les rites annuels de la maison et des communautés locales en transformation. Intérieur et extérieur des lieux de vie communautaires", s'attache à aborder les formes domestiques des cultes et leurs évolutions récentes dans leur relation avec les cultes locaux organisés par des confréries, en particulier dans différents sanctuaires. À la suite d'un état de la question, il s'agit d'un exposé très bien documenté sur l'état actuel des pratiques et les changements qui les affectent: banalisation ou sécularisation de certains aspects des rites, passage de la sphère domestique à la communauté, ou le contraire.

10 Kanda Yoriko enfin, dans sa contribution « Femmes de Sasaguri. Au fond du religieux et à l'ubac du séculier ", présente d'abord un tableau global de l'activité des femmes dans la région, avec deux registres principaux, celui de la mine et celui du religieux, et un domaine plus marginal et plus inattendu, celui de la prostitution. Le texte se présente ensuite comme une suite de portraits de femmes dont le destin illustre les carrières féminines les plus typiques. Le retour en fin d'article, après la longue évocation des rôles religieux, aux questions du travail minier et de la prostitution, surprend un peu, même si la coprésence des deux thèmes est justifiée par la place importante des mineurs parmi les dévots des temples considérés en raison des risques auxquels ils sont exposés.

$11 \mathrm{Au}$ total, il nous apparaît que la description précise d'une ville japonaise, certes " ordinaire ", mais aussi dotée avec le "nouveau pèlerinage de Shikoku à Sasaguri » d'un site religieux faisant l'objet de pratiques spécifiques, est une très bonne manière de pénétrer dans ce que le religieux au Japon a sans doute de plus déroutant pour un Occidental dont les conceptions sont plus ou moins modelées par la tradition chrétienne. On y découvre en effet un polythéisme actif, vivant, issu de traditions anciennes, mais aussi adapté à des formes cultuelles typiques de la modernité 
religieuse : comme si le cadre ancien se prêtait déjà à une sorte de "religion à la carte " typique de notre temps. On y rencontre aussi un foisonnement de spécialistes religieux qui, eux aussi, savent jouer avec les attentes de leur public et développer une offre très diversifiée, structurée par un système socio-économique qui évoque la petite entreprise familiale. À cet égard encore, le sol traditionnel des rôles religieux, déjà marqués par la large autonomie et la créativité des spécialistes, engendre sans rupture manifeste une foule de maîtres spirituels comparables à ceux qui existent aujourd'hui un peu partout dans le monde. La lecture de cette monographie exceptionnelle est ainsi une occasion de mesurer à la fois la proximité et la distance d'un Japon qui semble à ce jour encore marquer la singularité de sa modernité. 